\title{
Should hair transplantation be performed in psoriasis?
}

\author{
Anand Kumar Vaggu, MD, Ratchathorn Panchaprateep, MD, Shobit Caroli, MBBS, Kulakarn Amonpattana, MD, Oravan Pathomvanich, MD, \\ Damkerng Pathomvanich, MD Bangkok, Thailand anand2derma@yahoo.co.in
}

\section{Introduction}

Psoriasis is a chronic, relapsing, inflammatory, papulosquamous skin disease. Its prevalence in different populations varies from $0.1 \%-11.8 \% .^{1}$ Hair restoration surgery in psoriatic skin is challenging and, to date, there are no prospective studies existing on this topic. Most surgeons defer surgical procedures on active psoriatic skin because they would like to avoid incising the affected skin for fear of aggravating the psoriatic lesions (Koebnerization) with subsequent poor wound healing and increased wound infection due to the colonization of Staphylococcus aureus in many psoriatic lesions. ${ }^{2}$ There are many studies in orthopedic and podiatric literatures regarding surgical procedures such as hip or knee joint replacement, osteotomies, arthroplasties, and joint manipulation with psoriatic arthritis. ${ }^{9-13}$ To the best of our knowledge, this is the first report where hair transplantation was performed in 7 cases of psoriasis.

\section{Objective}

Our objective was to study the risk of eliciting the Koebner phenomenon of infection at both donor and recipient areas, and of poor wound healing in the donor area in psoriasis patients. At the same time, we set out to observe whether the psoriasis would become active at the recipient site from the grafts prepared from psoriatic lesions in the donor area.

\section{Material and Methods}

We performed hair transplantation on 7 patients with psoriasis: 6 cases for scalp transplantation and 1 for an eyelash transplant. In all cases, "ultra-refined" follicular unit grafting was done using a strip technique and trichophytic closure after obtaining consent and giving prior counseling about the Koebner phenomenon. In all patients, the donor strip was harvested by an open technique with four skin hooks. Tumescent fluid containing normal saline, epinephrine, and triamcinolone was injected over the recipient site in all the cases. Premade incisions and stick-and-place techniques with hypodermic needles were used to place the grafts. Perioperative antibiotics were given for all patients. Diazepam and Midazolam were used to sedate the patients. Either a paracetamol and codeine or a paracetamol and tramadol combination was given postoperatively. Demographics and transplant procedures are illustrated in Table 1. In the first case, we intentionally transplanted 525 follicular unit grafts on right frontal area (Figure 1B), which were prepared from a psoriatic lesion in the donor area (Figure 1A). In the same case, a second session was done 9 months after first transplant without any adverse consequences.

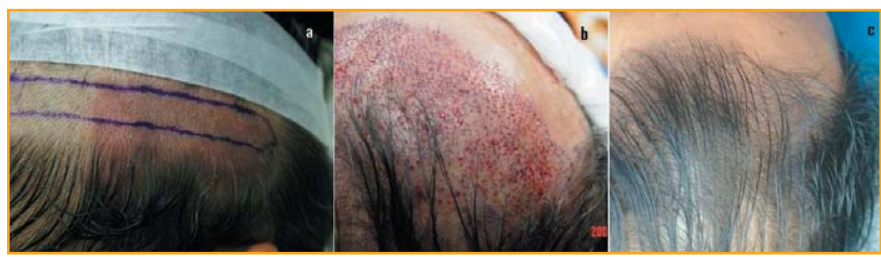

Figure 1. Case 1. A: Ill-defined psoriatic plaque incorporated in donor area. $B$ : 512 grafts prepared from psoriatic lesion were transplanted on right frontal area. $C$ : Excellent growth of grafted follicles after 9 months.

\begin{tabular}{|c|l|l|l|c|c|c|c|c|c|c|}
\hline $\begin{array}{c}\text { Case } \\
\text { No. }\end{array}$ & Age/Sex & Race & Diagnosis & $\begin{array}{c}\text { HT } \\
\text { session }\end{array}$ & $\begin{array}{c}\text { Total } \\
\text { grafts }\end{array}$ & $\begin{array}{c}\text { 1h } \\
\text { grafts }\end{array}$ & $\begin{array}{c}\text { 2h } \\
\text { grafts }\end{array}$ & $\begin{array}{c}\text { 3h } \\
\text { grafts }\end{array}$ & $\begin{array}{c}\text { Total } \\
\text { hairs }\end{array}$ & $\begin{array}{c}\text { Duration } \\
\text { since } \\
\text { transplant }\end{array}$ \\
\hline Case1 & $57 / \mathrm{M}$ & Asian & AGA III & 1 & 2500 & 469 & 1919 & 12 & 4343 & \\
\hline & & & & 2 & 1647 & 584 & 1032 & 25 & 1647 & $2 \frac{1}{2}$ years \\
\hline Case2 & $57 / \mathrm{M}$ & Caucasian & AGA VI & 1 & 3578 & 386 & 2606 & 586 & 7369 & 3 months \\
\hline case3 & $58 / \mathrm{M}$ & Caucasian & AGA V & 1 & 3361 & 597 & 2394 & 370 & 6495 & 10 months \\
\hline Case4 & $52 / \mathrm{M}$ & Caucasian & AGA VI & 4 & 1679 & 359 & 1081 & 239 & 3238 & 3 months \\
\hline case5 & $40 / \mathrm{M}$ & Caucasian & AGA III & 1 & 2777 & 351 & 1741 & 685 & 5888 & 1 month \\
\hline Case6 & $40 / \mathrm{M}$ & Asian & AGA IIIV & 1 & 2654 & 555 & 1607 & 494 & 5251 & 10 months \\
\hline Case7 & $25 / \mathrm{F}$ & Asian & Eye lash & 1 & 77 & 77 & & & 77 & $11 / 2$ years \\
\hline
\end{tabular}

Table 1. Demographics and transplant procedures

In the second case, psoriatic lesions in the donor area (Figure $2 \mathrm{~A} \& \mathrm{~B}$ ) were treated with topical clobetasol propionate $0.05 \%$ lotion for 2 weeks followed by application of Betamethasone dipropionate and calcipotriol ointment daily for 2 more weeks then only on weekends for maintenance. The patient came for transplantation after 3 months. By this time, all the lesions were improved except for mild erythema and scaling (Figure 2C \& D). In the third case, a $1.5 \times 2.5 \mathrm{~cm}^{2}$ psoriatic lesion was avoided during donor harvesting and the incision was taken just above the lesion (Figure 3A \& B). In the fourth case, a few ill-defined, erythematous patches were included in the donor strip. In the remaining three cases, no lesions were seen in the donor area. In the fifth case, in addition to a strip harvest, 169 FUE grafts from the scalp and 4 FUE grafts from the beard were used for a total of 1,679 FUGs consisting of 3,238 hairs. This patient had three transplants previously at another clinic without any aggravation of his psoriasis. In the sixth case, the patient had previously used methotrexate $10 \mathrm{mg} /$ week and stopped the medication 3 months prior to the transplantation.

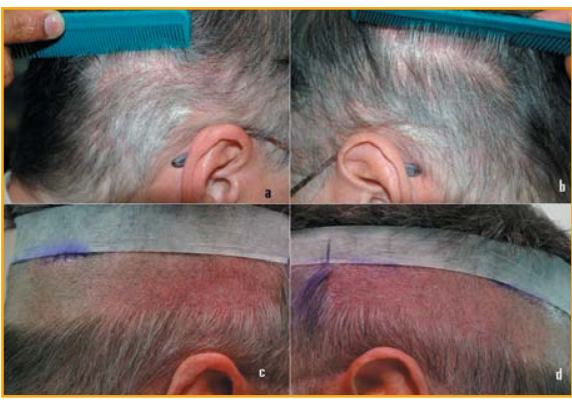

Figure 2. Case 2. $A \& B$ : Psoriatic plaques at donor area. $C \&$ $D$ : Mild erythema with fine scaling 3 months after treatment with topical medication.

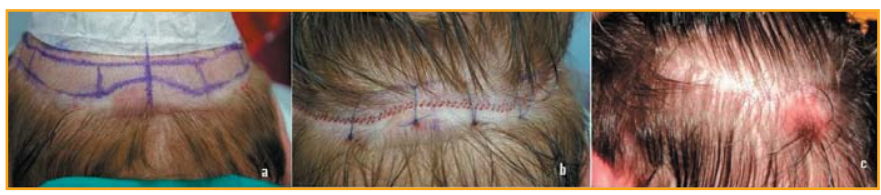

Figure 3. Case 3. A: Psoriatic plaque was avoided during donor harvesting. $B$ : Immediately after donor closure. $C$ : Normal healing over donor area after 6 months.

In all cases, mild to moderate psoriatic lesions were present on the trunk (Figure 4C) but not on the recipient area. All patients had been using only topical steroids by the time of transplantation. These cases have been followed up prospectively for the Koebner phenomenon, for aggravation of psoriasis, for infection at both the donor and recipient areas, and for poor wound healing in the donor area. Follow-up of each patient is shown in Table 1. 


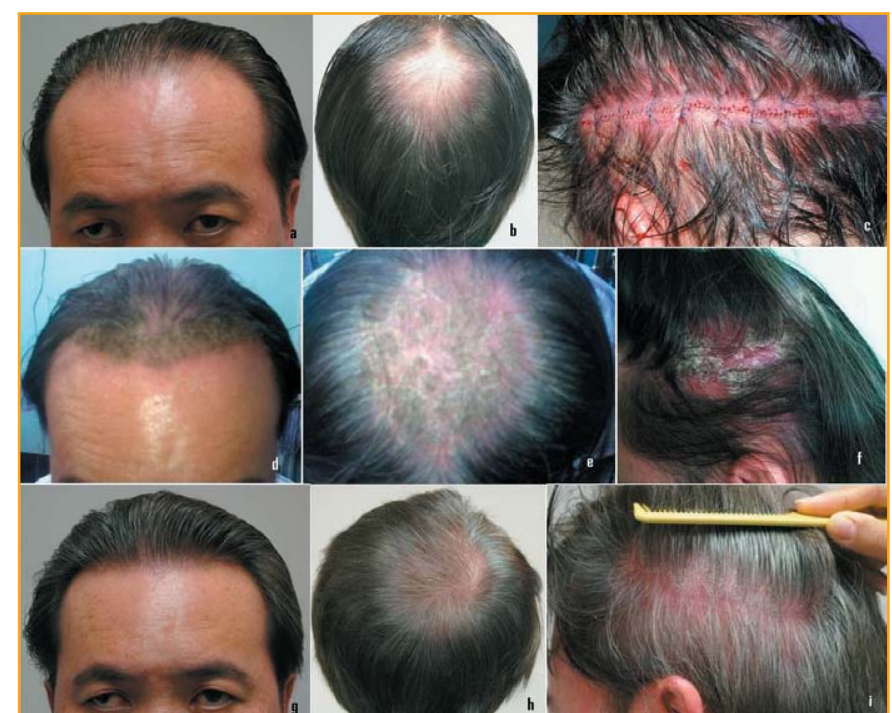

Figure 4. Case 6. $A \& B$ : Preoperative-Norwood class III vertex. $C$ : Immediately after donor closure. No psoriatic lesions present at both donor and recipient areas. $D, E, \& F$ Erythema, pustules, and crusting developed over both donor and recipient areas. (These photographs were mailed by the patient.) $G \& H$ : Excellent growth of transplanted hair at frontal area and vertex. I: Psoriatic lesions developed (Koebnerization) over left parieta area and also along the scar.

\section{Results}

Out of 7 cases, 6 patients have chronic plaque psoriasis and one patient has palmar psoriasis. In the first 5 cases of scalp hair transplantation, there was no aggravation of psoriasis at either the donor (Figure 3C) or the recipient area (Figure 1C) even though, in the first case, grafts were prepared from a psoriatic lesion. In the sixth case of scalp hair transplantation where follicular unit grafts were prepared from normal donor area (Figure 4C), the patient developed erythema, pustular lesions, crusting, and aggravation of his psoriasis over both the donor and recipient areas 3 weeks after his surgery (Figure 4D, E, \& F). A dermatologist confirmed it to be a pustular psoriasis and started Methotrexate $15 \mathrm{mg} /$ week. Within two weeks, all the lesions started drying up and subsided in one month. At 9 month follow-up, excellent growth of transplanted hair was noticed (Figure 4G \& H), but he developed chronic plaque psoriasis over the right and left parietal areas (Figure 4I). Presently, he is using methotrexate, topical clobetasol, 17-propionate $0.05 \%$ lotion, and Coal tar shampoo. This is the only case in which the psoriatic lesions were absent on both the donor and recipient areas before transplantation (Figure 4A, B, \& C) but developed Koebnerization after transplantation. In the eyelash transplant case, the post-operative period was uneventful and did not develop any psoriatic lesions along the lid margins (Figure 5). None of the patients presented with infection or poor wound healing in the donor area.

\section{Discussion}

Psoriasis is a common, chronic, inflammatory, proliferative, recurrent, papulosquamous condition that involves the skin and

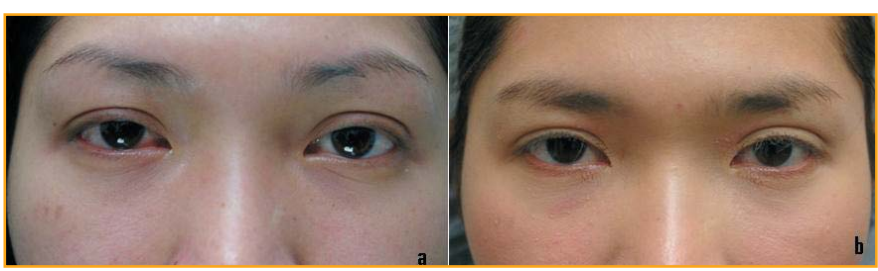

Figure 5. Case 7. A: Pre-operative-sparse eyelashes. $B$ : Post-operative $1 \frac{1 / 2}{2}$ years. sometimes the joints. Both genetic and environmental influences have a critical role. The most characteristic lesions consist of erythematous plaques covered by silvery white scales and show predilection for extensor joint surfaces and the scalp. Psoriasis may present an unpredictable course, with spontaneous improvement or exacerbation of the lesions. ${ }^{3}$ Psoriatic arthritis develops in approximately $10 \%-15 \%$ of those with psoriasis such as enthesopathy with proliferative arthritis of small and large joints, sacroilitis, spondyloarthritis, or synovitis. ${ }^{1}$ Psoriasis is one of the dermatological conditions in which the Koebner phenomenon is seen. The Koebner phenomenon is a development of similar psoriatic lesions in previously uninvolved skin of the patients from various types of trauma. This reaction usually occurs 7-14 days after injury, but it may range from 3 days to several years following injury, ${ }^{4}$ and the reported incidence varies between $38 \%-76 \%$ of patients with psoriasis. ${ }^{3}$ The clearing of existing psoriasis following injury has been observed and has been termed the "reverse Koebner reaction." The Koebner and reverse Koebner reactions do not coexist at the same time. ${ }^{3}$ The Koebner response is more commonly seen when psoriasis is unstable or flaring and less common in quiescent or resolving psoriasis. The Koebner phenomenon signifies activity of the disease. It warns the clinician to avoid physical/chemical irritants and surgical procedures, and to treat underlying or concurrent diseases in order to get good results. ${ }^{4}$ This reaction can occur in distant wounds of the patients with pre-existing cutaneous diseases, though it occurs most frequently in patients with psoriasis. Therefore, surgeons should be aware of this entity and should warn their patients about its possible occurrence. ${ }^{5}$ However, other concerns are the increased infection and poor wound healing in psoriasis. The skin of the psoriatic patient contains a higher bacterial count compared to the skin of normal people. ${ }^{15}$ Marples et al. found that psoriatic lesions were colonized with Staphylococcus aureus $46 \%$ of the time, while the skin in unaffected areas yielded S.aureus $28 \%$ of the time. ${ }^{6}$ Noble states in his study that Staphylococcus is more common on the skin of patients with skin diseases, including psoriasis. ${ }^{7}$ Apart from this, surgical stress and drugs such as NSAIDS can aggravate psoriasis. Emotional stress, alcohol use, smoking, obesity, and lack of exercise have been found to adversely exacerbate psoriasis. ${ }^{14}$

Shupuck and Saini did a survey on the practices and beliefs of dermatologists, orthopedic surgeons, and plastic surgeons. ${ }^{2}$ Out of 65 dermatologists who performed surgery on active psoriatic skin, 14 dermatologists experienced aggravation of psoriasis and 2 dermatologists witnessed delayed wound healing. Twenty-five orthopedists out of 77 experienced complications with the most frequent one being wound dehiscence (12 cases) and second most common being wound infection ( 9 cases). Out of 23 plastic surgeons having experience in performing surgery in active psoriatic skin, only 2 witnessed delayed wound healing. Lambert and Wright found a very low rate of infection in their study on psoriasis and arthritic patients who underwent various surgical procedures (2 out of 54), including orthopaedic surgeries (1 out of 41). ${ }^{9}$ However, studies of total hip and knee arthroplasty in psoriasis patients have reported increased infection rates. Menon \& Wroblewski reported 9.1\% superficial and $5.5 \%$ deep infection rates (compared to $7.4 \%$ and $1.2 \%$, respectively, in normal populations) in patients with psoriasis undergoing Charnley total hip arthroplasty without the use of 
Psoriasis

from page 125

perioperative antibiotics. Stern et al. demonstrated an overall deep infection rate of $17 \%$ (compared with $0.8 \%$ to $1.9 \%$ seen in nonpsoriatics) due to Staphylococcus and beta-hemolytic Streptococcus organisms in psoriatic patients even when they received peri-operative antibiotics, but they also included rheumatoid arthritis patients in their study. ${ }^{11}$ In another study of 50 primary total knee arthroplasties performed on 34 patients with psoriasis vulgaris, only one deep infection (2\%) with S.aureus occurred 25 months after operation. There were no immediate post-operative wound infections either superficial or deep. No one had impaired wound healing but exacerbation of psoriasis was noted in 2 patients. ${ }^{12}$ However, the authors suggested that the use of prophylactic antibiotic therapy may reduce these rates significantly. Ryan reported a case of 30-year-old female who underwent distal interphalangeal joint arthrodeses for advanced psoriatic arthritis of both hands and 2 weeks later developed psoriatic lesions beneath the splint. ${ }^{13}$ Koebnerization should not be alarming because psoriatic skin is known to heal at a normal rate, and, after completion of healing, it can be treated with the topical dermatologic regimens. ${ }^{8}$ In the study of the surgical management of psoriasis, psoriatic lesions developed along the margin of the shaved area in 7 patients after serial dermatome shaving of psoriatic plaques to the middle of the reticular dermis in 24 patients. ${ }^{16}$ Nottrott et al. reported a case of a wide resection of an extra-skeletal chondrosarcoma of the thigh and reconstruction with a femoral megaprosthesis involving an area of psoriasis that, with special emphasis on post-operative wound healing, did not have any post-operative problems. ${ }^{15}$

Most of the previous anecdotal responses and retrospective studies were related to orthopedic and podiatric surgery with no prospective studies with hair transplantation in psoriasis. To the best of our knowledge, this is the first prospective study on hair transplantation in psoriasis. Eisenberg reported one patient who developed psoriasis in the transplanted area.${ }^{17}$ In our study, except in 1 of the 7 patients, none developed Koebnerization. No infections occurred in either the donor or the recipient areas. Normal healing occurred in the donor area in all the patients, even in the patient where the skin incision was placed at the edge of psoriatic plaque. We didn't encounter any complication resulting from incorporating the psoriatic lesion in the donor strip and also the transplanted grafts prepared from the lesion showed excellent growth. The risk of Koebnerization is very low, lower than we anticipated. This could be because all of our cases had mild and subsiding psoriasis. As seen in one case, if new lesions do occur, treatment with methotrexate resulted in resolution of the lesions without any adverse effect on the outcome of the transplanted hair. Shiell stated, anecdotally, that all the lost hair returned back in his psoriasis patient even after a violent reaction with massive encrustation after hair transplantation. ${ }^{18}$ If any psoriatic lesion is present in the donor area, it is prudent to treat at least 2 weeks prior to hair transplantation. In contrast to reported occurrences of wound infection in orthopedic studies, we did not notice any infection. The reason could be hair transplantation is a superficial surgical procedure and the scalp is richly vascularized. However, apart from incision trauma, surgical stress can also aggravate psoriasis as it occurs on an individualized basis. Therefore, this phenomenon should be kept in mind in every psoriasis case and the patient should be advised of the possible risk of these problems before the surgery.

\section{Conclusion}

Hair transplantation can be performed on psoriasis patients with prior counseling of the possibility of the Koebner phenomenon. Even if it occurs, it will not affect adversely the hair transplantation outcome with institution of proper dermatological treatment.

\section{References}

1. Klaus, W., et al. Fitzpatrick's Dermatology in General Medicine, 7th Ed. New York: McGraw-Hill, 2007; 169-207.

2. Saini, R., and J.L. Shupack. Psoriasis: to cut or not to cut, what say you? Dermatol Surg. 2003; 29:735-740.

3. Burns, T., et al. Rook's Textbook of Dermatology, 8th Ed. Wiley-Blackwell, 2010; 20.1-20.60.

4. Thappa, D.M. The isomorphic phenomenon of Koebner. Indian J Dermatol Venereol Leprol. 2004; 70:187-189.

5. Mendez-Fernandez, M.A. Koebner phenomenon: what you don't know may hurt you. Ann Plast Surg. 2000; 44:644645.

6. Marples, R.R., C.L. Heaton, and A.M. Kligman. Staphylococcus aureus in psoriasis. Arch Dermatol. 1973; 107(4):568-570.

7. Noble, W.C. Distribution of the microccaceae. Br J Dermatol. 1969; 81:27-32..

8. Lynfield, Y.L., G. Ostroff, and J. Abraham. Bacteria, skin sterilization, and wound healing in psoriasis. $N Y$ State $J$ Med. 1972; 72:1247-1250.

9. Lambert, J.R., and V. Wright. Surgery in patients with psoriasis and arthritis. Rheumatol Rehabil. 1979; 18:35.

10. Menon, T.J., and B.M. Wroblewski. Charnley low-friction arthroplasty in patients with psoriasis. Clin Orthop Relat Res. 1989; 176:127-128.

11. Stern, S.H., et al. Total knee arthroplasty in patients with psoriasis. Clin Orthop Relat Res. 1989(Nov); 248:108-111.

12. Beyer, C.A., et al. Primary total knee arthroplasty in patients with psoriasis. J Bone Joint Surg. 1991; 73:258-259.

13. Ryan, G.M. Psoriatic arthritis and Koebner phenomenon [letter]. J Hand Surg. 1991; 16A:180-181.

14. Raychaudhuri, S. Psoriasis risk factors: role of lifestyle practices. Cutis. 2000; 66: 348-352.

15. Markus, N., et al. Wound healing in a patient with psoriasis vulgaris and femur megaprosthesis implantation. Sarcoma. Article ID 509242, 3 pages, 2008.

16. Drancourt, M., et al. Psoriasis is a risk factor for hip prosthesis infection. Eur J Epidemiol. 1997(Feb); 13(2):205-207.

17. Eisenberg, E. In: Hair Transplantation, 4th Ed. W.P. Unger and R.S. Shapiro, eds. New York: Marcel Decker; 78.

18. Shiell, R. Transplanting in the psoriatic patient. [Cyberspace Chat] Hair Transplant Forum Int'l. 2006; 16(6):223. 